\title{
THE IMPACT OF OSCILLATOR STRENGTH ON SILICON NANOCRYSTALS (SiNCS)
}

\author{
Ishaya Iliyasu, Abbas Dalhatu Umar, Abubakar Yusuf, Aliyu Muhammad, Williams Danbaba \\ Department of Science lab. tech \\ Nuhu Bamalli Polytechnic, Zaria, Kaduna State, Nigeria
}

\begin{abstract}
A theoretical simulation is used to estimate the impact of oscillator strength on Silicon nanocrystals (SiNCs) using Matlab program. The Photoluminescence intensity oscillator dependence as well as Oscillator strength as a function of energy band gap and photon energy is studied. The results obtained, shows that oscillator strength of SiNCs behavior is in compliance with the quantum confinement effects which in succession determine the optical properties of SiNCs, and also shows that the oscillator strength in Nano-Crystallites varies with the amount of confinement and also with the surrounding environment due to change in dielectric constant. The models used in this work can be applied to other nanocrystals semiconductor materials
\end{abstract}

Keywords - Nanocrystals, Matlab, Oscillator strength, Silicon, bandgap.

\section{INTRODUCTION}

Silicon is one of the most abundant elements of the earth crust after oxygen. The whole semiconductor industry is based on silicon because of its chemical and physical properties. Towards the end of the 20th century it became clear that properties of silicon change in the nanoscale in a way that these changes enable coupling visible light photons with electrons of silicon. Thus Nano sized silicon based systems promise to the semiconductor industry the chance of low cost fabrication of interesting devices such as tunable lasers, light emitting diodes, non-volatile memories. [1]

Being the most widely spread semiconductor has led to its overcoming dominance in modern microelectronic devices. However, building optoelectronic devices is impossible with bulk silicon due to its indirect band gap. SINCs band gap increases with decrease in size and photoluminescence (PL) with an obtained external efficiency in excess of about $12 \%$ [1]. which can be attributed to the radiative recombination of electrons and holes in the quantum confined nanostructure [2]

Quantum confinement effects play an important role in optical absorption and luminescence semiconductor [3]. In addition to quantum confinement increases the oscillator strength in a semiconductor nanostructure compared to the bulk state, making materials like silicon viable for optoelectronics [4]. To compare the transition strength between different types of quantum mechanical systems the oscillator strength is a useful method. The oscillator strength is a dimensionless quantity that expresses the probability of absorption or emission of electromagnetic radiation in transition between energy levels of an atom or molecule $[4,5]$ The oscillator strength can be thought of as the ratio between the quantum mechanical transition rate and the classical absorption/emission rate of a single electron oscillator with the same frequency as the transition [6]. Cosputtering, ion implantation, chemical vapor deposition (CVD), molecular epitaxy and laser ablation methods have been used to fabricate SiNCs. $[7,8,9,10]$

The main objective of this work is to study. The theoretical models used to find the influence of oscillator strength on optical behavior of silicon nanocrystals using Matlab simulation.

\section{METHOD}

The theoretical simulation considered in this work allows one to observe the effect of oscillator strength on silicon nanocrystals from measured quantities such as the photon energy, the energy band gap and the photoluminescence intensity. A model of photoluminescence intensity as a function of quantum confinement, oscillator strength is investigated in the silicon nanocrystals (SiNCs).

The quantum confinement increases the oscillator strength and is based on the electronic confinement in the nanostructure. The size dependence of the optical band gap of nanostructure is given by

$$
E_{g}^{\text {nano }}=E_{g}^{\text {bulk }}+\frac{\beta}{d Y}---(1)
$$

Where $\beta$ and $\gamma$ are the quantum confinement parameters, $E_{g}^{\text {nano }}{ }_{n} E_{g}^{\text {bulk }}$ and $d(n m)$ are the band energy of silicon nanocrystals, bulk silicon band gap energy at room temperature and diameter (size) of the silicon nanocrystal respectively.

The values of the energy gap and diameter were then extracted from the experimental work of Hong et al (2011) presented in Fig 1 below using GetData graph Digitizer software and substituting in equation (1) above to get the quantum confinement parameters. 


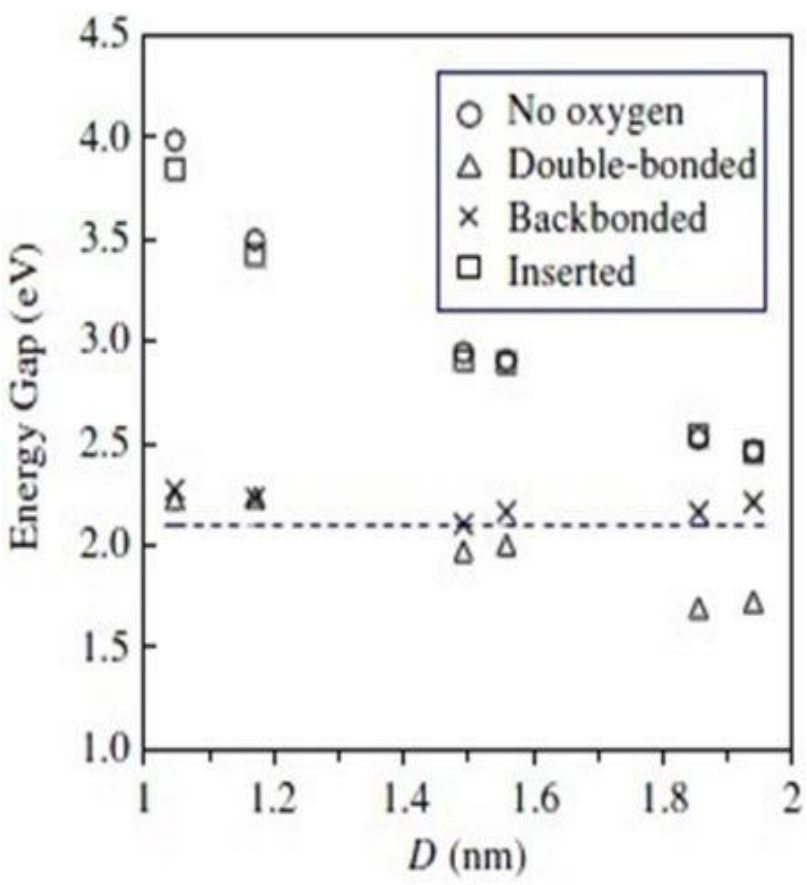

Figure 1: Energy gap versus Diameter. Hong et al[9]

The concept of oscillator strength is understood by studying the matrix elements this also provides insight into electronics transition rules. Considering electromagnetic field of sufficiently short wavelength, this initiates electronic transitions from valence band to conduction band.

$\vec{E}=A_{0}, \hat{\theta}_{x} \exp \left\{i\left(k_{0} r-\omega t\right)\right\}---(2)$

Where $A_{0}=$ Amplitude, $\hat{\theta}=$ electronic polarization, $k_{0}=$ wave vector of the light travelling in r-direction.

The number of optically induced transitions at the same $\mathrm{k}$ between band $\mu$ and $\nu$ is proportional to the square of momentum matrix elements given by

$e_{x} M_{\mu V}(k)=\left\langle\varphi_{\mu}|\hat{e} \rho| \varphi_{V}\right\rangle---(3)$

The matrix elements can be approximated from Kane-estimate using

$\left|e_{.} M_{\mu \nu}\right|^{2}=\left(\frac{m_{0}}{\hbar}\right)^{2} P^{2}---(4)$

Where, $\mathrm{P}$ is the momentum matrix parameter. Kane estimated that

$P^{2}=\frac{3}{2} \hbar^{2}\left(\frac{m_{0}-m_{e}^{*}}{m_{0} m_{\theta}^{*}}\right) \frac{E_{g}-\Delta_{0}}{3 E_{g}+2 \Delta_{0}} E_{g}---(5)$

Where, $\Delta_{0}$-spin orbit splitting energy, $m_{\theta}^{*}$-effective mass of electron in the conduction band, $E_{q}$ - band gap, $m_{0}-$ electron rest mass.

The matrix element will be $\left|e_{.} M_{\mu \nu}\right|^{2}=\frac{3}{2} \hbar^{2}\left(\frac{m_{0}-m_{\theta}^{*}}{m_{0} m_{\theta}^{*}}\right) \frac{E_{g}-\Delta_{0}}{3 E_{g}+2 \Delta_{0}} E_{g}---(6)$

This relates the band gap with the matrix element.

The oscillator, the complement number of oscillations of the changeover between the valence and conduction bands is related to the matrix element of the momentum matrix $P_{C V}$ by

$f_{C D}=\frac{2\left|P_{C V}\right|^{2}}{m_{0} \hbar \omega_{C \theta}}---(7)$

Where $P_{C D}=\left|\hat{e} M_{C V}\right|$

This implies that

$$
f_{C V}=\frac{2\left|\theta M_{\mu v}\right|^{2}}{a m_{Q} \hbar w_{e v}}---(8)
$$

The factor $1 / 3$ in equation (8) is due to the averaging with $\left|M_{x}\right|^{2}=\left|M_{y}\right|^{2}=\left|M_{z}\right|^{2}=\frac{|M|^{2}}{2}$

Insert equation (6) into (8) and using $m_{e}^{*}=0.4 m_{0}$ we obtain

$f_{C V}=\frac{2}{3 m_{0} \hbar \omega_{C V}} \frac{3}{2} \frac{m_{0}}{m_{\theta}^{*}}\left(m_{0}-m_{\theta}^{*}\right) \frac{E_{g}+\Delta_{0}}{3 E_{g}+2 \Delta_{0}}---(9)$

$f_{C V}=\frac{3}{2 m_{0} \hbar \omega_{c v}} \frac{E_{g}+\Delta_{0}}{3 E_{g}+2 \Delta_{0}} E_{g}--(10)$

This derived expression can then be estimated using Matlab program $[6,9]$

From equation (10) depend on quantum band gap and photon energy. The general expression for photoluminescence intensity for nanostructures used is given as

$I(\Delta E)=\frac{1}{(\sigma \sqrt{2 \pi})}\left(\frac{\beta}{\Delta E^{2}}\right)^{\frac{\sigma-\alpha+\gamma}{\gamma}} \exp \left\{\frac{-\left[\left(\frac{\beta}{\Delta E}\right)^{\frac{1}{\gamma}}-L_{0}\right]^{2}}{2 \sigma^{2}}\right\}-\cdots$

\section{EXPERIMENT AND RESULT}

The result of the fitted band gap against size(nm) is presented I Fig 2 which was obtained from fitting with the experimental work of Hong Yu et al [2,3,9]. Which is represented by the encircled points while the blue line represents the full line graph with the quantum parameters of $\beta=3.04 \mathrm{eV}$ and $\gamma=1.26$ 


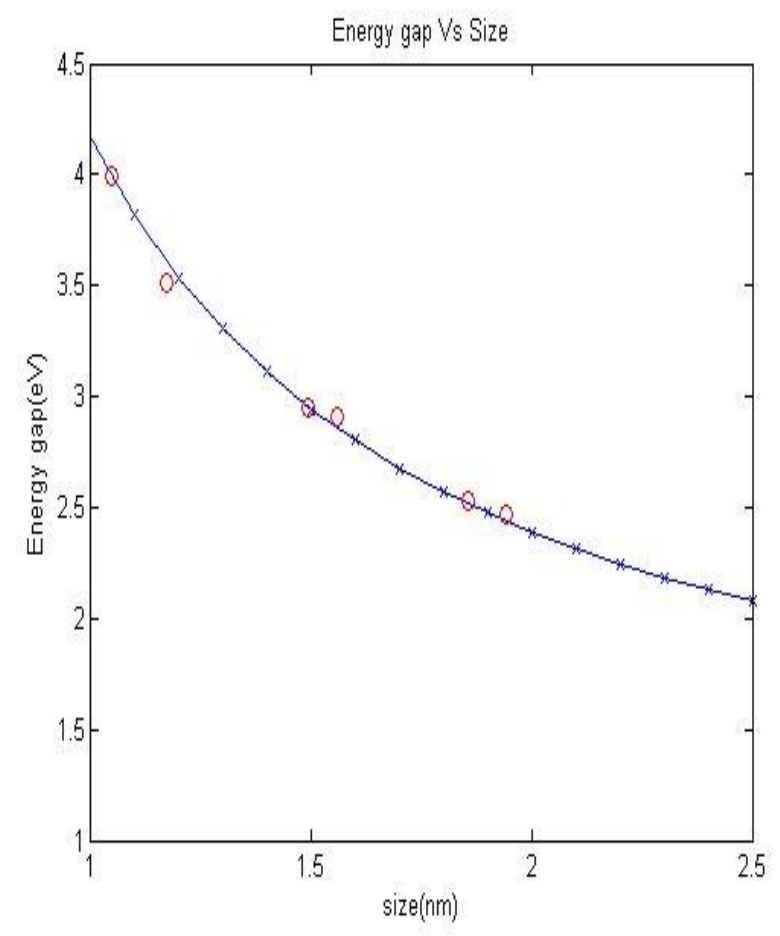

Fig 2: BandGap energy as a function of size

From the obtained quantum parameters equation, one becomes

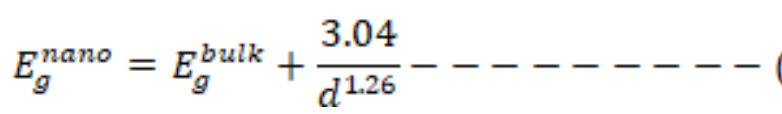

Using equations (12) and (10) the plot of the oscillator strength versus effective size ranging from $(0.5-14 \mathrm{~nm})$ for silicon nanocrystals at a given photon energy $(2.2 \mathrm{eV})$ with spin orbit splitting of $\left(\Delta_{0}=0.044\right)$, electron mass of $\left(m_{0}=0.72\right)$ is shown I fig 3 .

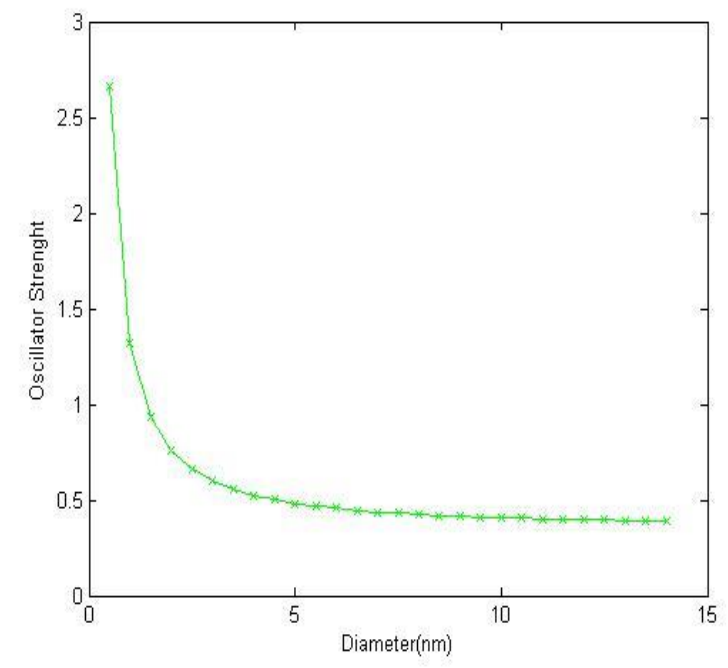

Fig3: Oscillator strength as a function of size
The plot of oscillatory strength as a function as a function photon energy range of $0.2-4.0 \mathrm{eV}$ for silicon nanocrystals of band energy $1.52 \mathrm{eV}$ is shown in Fig 4.

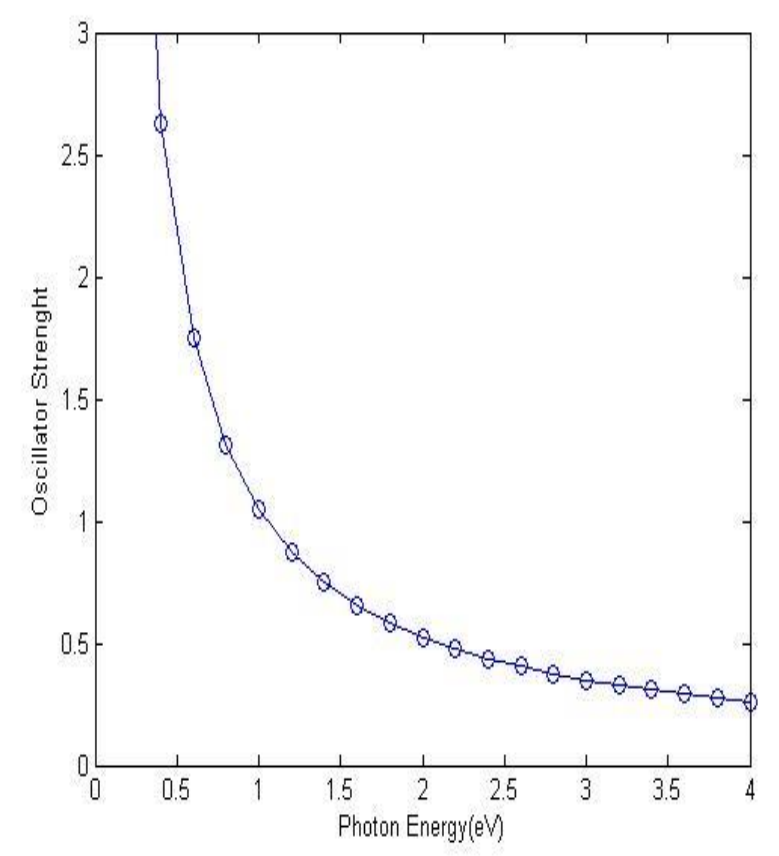

Fig 4: Oscillator strength as a function of photon energy

It is found that the oscillator strength decreases for increasing nanoparticle size and photon energy for silicon nanoparticles. So, the oscillator strength of silicon nanocrystals is shown to depend strongly on the size of the nanocrystals. A simulation by Anley [9] to study the oscillator strength shown in fig 5 and Fig 6 and our result is in agreement. 


\section{International Journal of Engineering Applied Sciences and Technology, 2020 \\ Vol. 5, Issue 8, ISSN No. 2455-2143, Pages 49-53 \\ Published Online December 2020 in IJEAST (http://www.ijeast.com)}

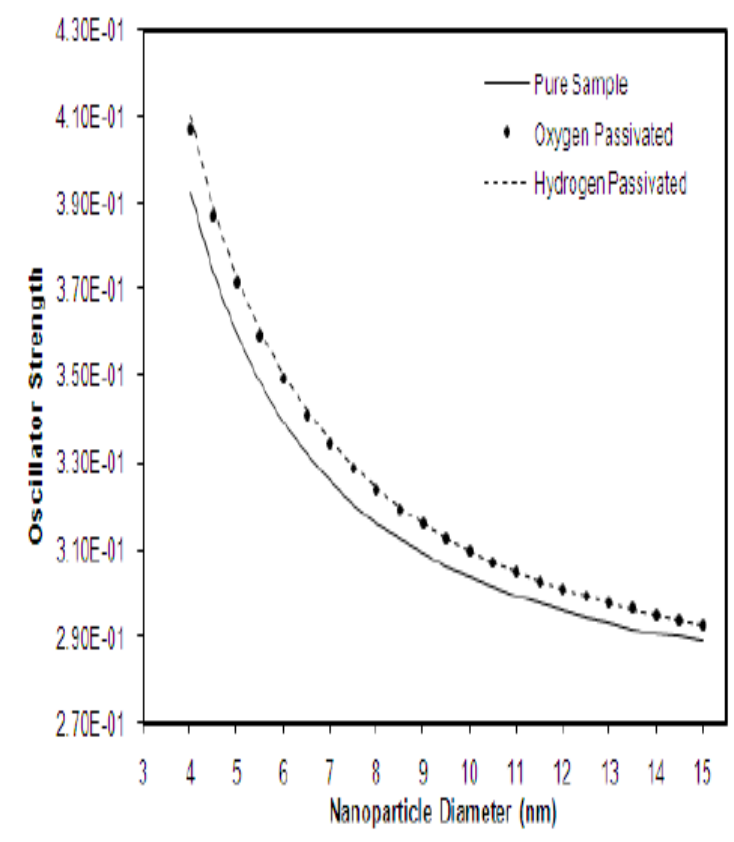

Fig5: Oscillator strength against size Anley, 2009[9].

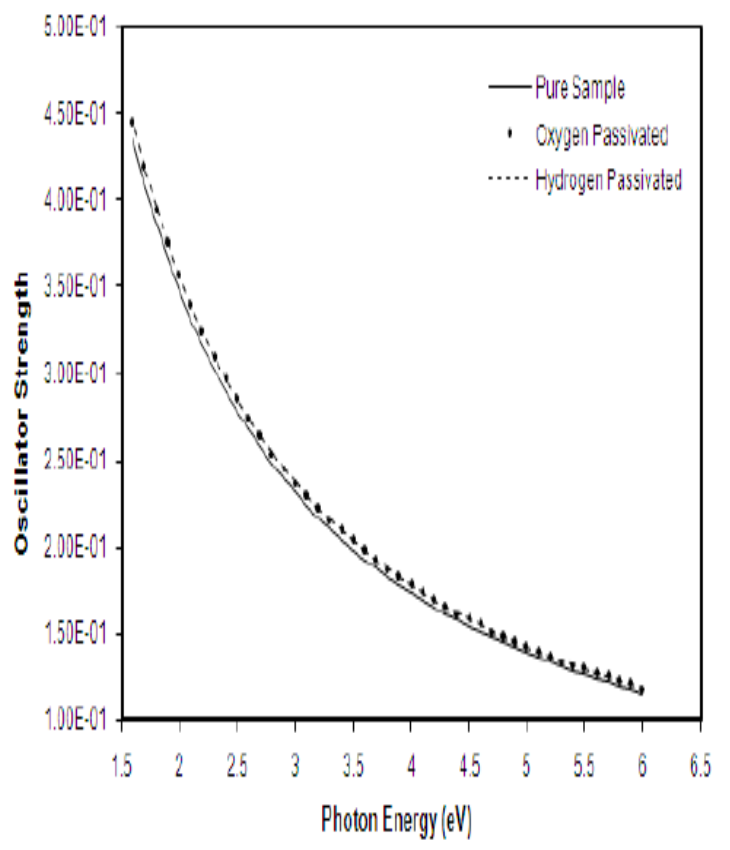

Fig 6: Oscillator strength against Photon energy of silicon nanocrystals. Anley, 2009 [9].

The model was used to simulate the Photoluminescence intensity against photon energy using MATLAB, fig 7 shows the result of the plot obtained which is line with the experimental work of [11]

\section{CONCLUSION}

In this work our aim is to study the influence of oscillator strength on silicon nanocrystals this was done by using a model and deriving the oscillator strength formula and integrating it with the quantum confinements. The results exhibit that by controlling a set of parameters extracted by fitting this developed model with experimental finding, it is possible to interpret the observed spectral features. The variation of oscillator strength as a function of silicon nanocrystals is presented and has shown great influence on the silicon nanocrystals which increases the relative transition strength as the band gap reduces to smaller size. It is shown how mean diameter and band gap energy of affects the oscillator strength. The importance of localized surface states in predicting the oscillator strength data from SiNCs using the developed model is demonstrated. We conclude that the oscillator strength of silicon nanocrystals strongly depends on the size of the nanocrystals i.e. on the quantum confinement but also on the surface passivation. Our results are in qualitative agreement with other observations as well as with experimental results.

\section{REFERENCE}

[1] Gelloz, B., Kojima, A., and Koshida, N. (2005)Highly efficient and stable luminescence of nanocrystalline porous silicon treated by highpressure water vapor annealing. Appl. Phys. Lett.87,031107. Doi:10.1063/1.2001136

[2] Aliyu,K.I., Ghoshal,S.K. (2015). Photoluminescence spectral features of silicon nanowires. Jurnal Teknologi. Vol. 78:3-2, PP. 153-158

[3] Ghoshal, S.K., Sahar, M.R., Arifin, R., Rohani, M.S., and Hamzah, K. (2014). Luminescence from silicon and germanium nanowires: A phenomenological model. Advance Material Research, 895, 424-428.

[4] Demtroder,W.(2003). "Laser Spectroscopy: Basic concept and instrumentation." Springer.p.31. ISBN 978-3-540-652-0.

[5] Kartopu,G., Bayliss,S.C., Hummel,R.E and Ekinci,Y. (2004). "Simultaneous micro-Raman and Photoluminescence study of spark-processed germaium: report oon th origin of Photoluminescence emission band.”J.Appl.Phys.95, 3466

[6] Anley,G.(2009). "Estimation of Absorption Coefficient, Oscillator Strength and Dielectric Function of Small Silicon Nanocrystallites" Master's thesis, Addis Ababa University, Addis Ababa, Ethiopia, PP. 32-33.

[7] Ghoshal, S. K., Gupta, U., and Gill, K. S. (2005). Role of Hydrogen and Oxygen on the Band Gap of Silicon Quantum Dots Indian Journal of Pure \& Applied Physics. 43: 188-191. 
[8] Bettotti,P., Cazzanell,M., Dal Negro,L., Anese,B.D., Gaburro, Z., Oton,C.J., Vijaya Prakash,G, and Pavesi,L.(2002).Silicon nanostructures for Photonics. J.phys:Condens matter Vol(14), pg 8253.

[9] Hong, Y., Jie-Qiong, Z., Zheng-Rong, Q. (2011)" silicon nanocrystals, crystalline Silicon-properties and uses. InTech, page 344.

[10] Proot, P., Delerue, C., and Allan, G. (1992). Electronics Structure and Optical Properties of Silicon Crystallites: Application to Porous Silicon. Applied Physics Letter. 61: 1948-1950.

[11] Ledoux. G., Guillois.O., Porterat. D., \& Reynaud. C., (2000). Photoluminescence properties of $\mathrm{Si}$ nanocrystals IOP publishing, doi: 10.1088/09574484/20/13/135702.

[12] Weng.D.,Jiong.Z.,Wenhao.Q.,Wei.Y.,Guangsheng.F (2008)'Dependance of the Photoluminescence from silicon nanostructures on the size of silicon nanoparticles' Optoelectronic Materials and Devices III;713508. Doi:10.1117/12.802285

[13] Thorsten.T.and Bardos.R (2005)'Photoluminescence: a surprisingly sensitive lifetime technique'. $31^{\text {st }}$ IEEE Photovoltaic Specialists conference. Pg (903-906) 